can generate about 1 volt during photosynthesis in the plant. Nathan Nelson and his colleagues at Tel Aviv University measured the electrical potential produced in crystals containing hundreds of layers of photosystem I placed on gold, silicon carbide, or indium tin oxide surfaces.

The complexes lined up head to toe, like batteries connected in series. The material produced up to 45 volts when illuminated with laser light, and also generated internal electric fields of up to 100 kilovolts per centimetre — among the strongest ever reported in a crystalline material, even among inorganic semiconductors.

The researchers say that the material could be used to make more efficient high-voltage optoelectronic devices.

Adv. Mater. http:/dx.doi.

org/10.1002/adma.201200039 (2012)

\section{Solar panel in the eye}

Special glasses that fire nearinfrared signals onto a device implanted into the retina could one day help to restore vision in blind people. This system would require fewer implanted components such as wires and coils to power the device than other proposed retinal prostheses.

James Loudin at Stanford

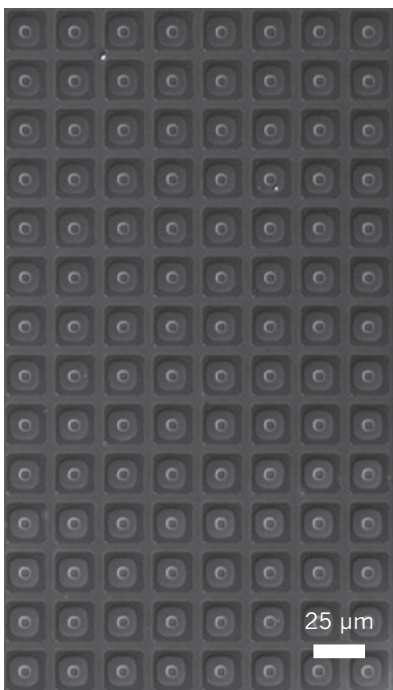

University in California and his colleagues designed arrays of photovoltaic diodes (pictured) that can be inserted into the retina and used to stimulate inner retinal cells. The diodes could ultimately form part of a system whereby a video camera attached to glasses would gather and feed image data to a small portable computer, which would then convert the data into near-infrared light pulses. The glasses would beam those pulses through the eye onto the implanted diodes. This light would deliver both visual information and power to the diodes.

The researchers showed that the photodiode array could activate cells in both healthy and degenerating rat retinas in vitro when pulsed with near-infrared light.

Nature Photon. http://dx.doi. org/10.1038/nphoton.2012.104 (2012)

\section{ASTRONOMY}

\section{Exoplanet signals ring true}

Most candidate multi-planet systems spotted by the Kepler space telescope probably contain true exoplanets, according to a statistical analysis.

Kepler spots potential planets beyond our Solar System by looking for tiny dips in brightness as the planets pass in front of their host stars. The method allows the telescope to monitor many stars at once, but can also give false-positive signals.

Assuming that false positives would be randomly distributed among the stars, Jack Lissauer at NASA's Ames Research Center in Moffett Field, California, and his team conducted a statistical test of where candidate planets identified by Kepler are located. They found that more than a third of possible candidates exist as part of multi-planet systems. This is higher than predicted by chance, suggesting that most of these systems contain true planets.

Astrophys. J. 750, 112 (2012)

COMMUNITY CHOICE

The most viewed papers in science

\title{
NEUROSCIENCE
}

\section{The neural core of consciousness}

\section{HIGHLY READ \\ on www.jneuro- sci.org in April}

'Waking up' from an unconscious state requires the activation of only primitive areas deep in the brain - not the higher cortical areas indicated in previous studies on anaesthetized people.

Harry Scheinin at the University of Turku in Finland and his colleagues used position emission tomography to image the brains of 20 volunteers recovering from anaesthesia. The participants had been given either propofol, a standard anaesthetic, or dexmedetomidine, an unusual sedative drug that allows the individual to be awoken temporarily with a shout or a prod. The two-drug study design allowed the researchers to differentiate between brain activities due to the drug and those specifically related to the conscious state.

They found that a core neural network involving just subcortical brain areas and a primitive part of the cortex was activated as subjects recovered sufficient consciousness to respond to verbal instructions.

J. Neurosci. 32, 4935-4943 (2012)

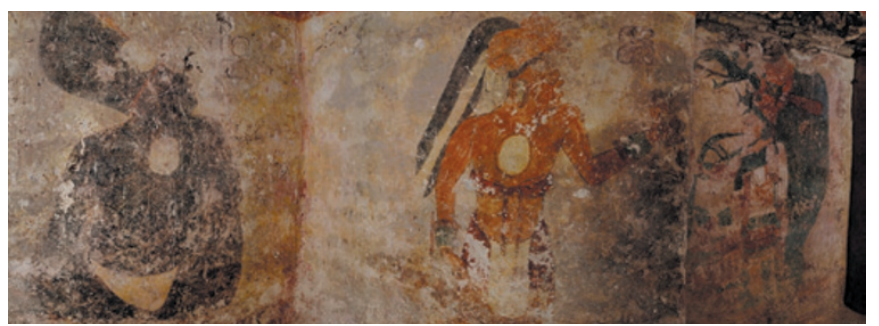

\section{ANTHROPOLOGY}

\section{Ancient Mayan wall calendar}

In an underground chamber in Guatemala, archaeologists have discovered the earliest evidence so far of Mayan astronomical tables: dates, numbers and depictions of lunar deities painted or carved on the walls some 1,200 years ago.

William Saturno at Boston University in Massachusetts and his colleagues stumbled across the paintings (pictured) while excavating the Mayan city of Xultun. On one wall the researchers found a table containing four columns of numbers, which could represent recurring events related to the cycles of Venus, the Moon, Mars and possibly Mercury. A table on another wall seemed to show 27 columns of dates, each 177 or 178 days apart, with Moon deities at the top of each column. The Maya recorded movements of the Moon in semesters of 177 and 178 days, or six lunar months.

Science 336, 714-717 (2012)

For a longer story on this research, see go.nature.com/ hlpigr

\section{CORRECTION}

The story 'Graphene's silicon cousin' (Nature 485, 9;

2012) should have said that silicon was deposited onto a silver surface heated to more than $200^{\circ} \mathrm{C}$. The silicon was heated to more than $1,000^{\circ} \mathrm{C}$.

\section{ONATURE.COM}

For the latest research published by Naturevisit:

www.nature.com/latestresearch 